\title{
Extensions of posets with an antitone involution to residuated structures
}

\author{
Ivan Chajda, Miroslav Kolařík and Helmut Länger
}

\begin{abstract}
We prove that every not necessarily bounded poset $\mathbf{P}=\left(P, \leq,^{\prime}\right)$ with an antitone involution can be extended to a residuated poset $\mathbb{E}(\mathbf{P})=(E(P), \leq, \odot, \rightarrow, 1)$ where $x^{\prime}=x \rightarrow 0$ for all $x \in P$. If $\mathbf{P}$ is a lattice with an antitone involution then $\mathbb{E}(\mathbf{P})$ is a lattice, too. We show that a poset can be extended to a residuated poset by means of a finite chain and that a Boolean algebra $\left(B, \vee, \wedge,{ }^{\prime}, p, q\right)$ can be extended to a residuated lattice $(Q, \vee, \wedge, \odot, \rightarrow, 1)$ by means of a finite chain in such a way that $x \odot y=x \wedge y$ and $x \rightarrow y=x^{\prime} \vee y$ for all $x, y \in B$.
\end{abstract}

\begin{abstract}
AMS Subject Classification: 03B52, 06A11, 06B05, 03B47
\end{abstract}
Keywords: Poset, bounded poset, residuated poset, residuated lattice, antitone involution, extension

Residuated posets in general and residuated lattices in particular form an algebraic axiomatization of certain substructural logics (see e.g. [4, 8, ,9] and [10] and references therein), especially of fuzzy logic, see [1] for details. Residuated lattices were studied for a long time starting with the pioneering paper by Ward and Dilworth [12], see also [3] and [7]. Posets and lattices with an antitone involution can serve as a suitable model of such a logic because this involution can be considered as a negation and hence these logics satisfy the double negation law, see [3]. Let us mention that a kind of residuated posets were studied also in [5]. Moreover, residuated structures derived from semirings were treated in [6] and [9].

Recall that a poset with an antitone involution is an ordered triple $\left(P, \leq,^{\prime}\right)$ such that $(P, \leq)$ is a poset and ' is a unary operation on $P$ satisfying

- if $a \leq b$ then $b^{\prime} \leq a^{\prime}$,

- $a^{\prime \prime} \approx a$

for all $a, b \in P$. Recall further that a residuated poset is an ordered quintuple $(P, \leq, \odot, \rightarrow$ ,1) such that

- $(P, \leq, 1)$ is a poset with a greatest element,

\footnotetext{
${ }^{1}$ Support of the research of the first and the third author by the Austrian Science Fund (FWF), project I 4579-N, and the Czech Science Foundation (GAČR), project 20-09869L, entitled "The many facets of orthomodularity", as well as by ÖAD, project CZ 02/2019, entitled "Function algebras and ordered structures related to logic and data fusion", and, concerning the first author, by IGA, project PřF 2020 014, is gratefully acknowledged.
} 
- $(P, \odot, 1)$ is a commutative monoid,

- $\rightarrow$ is a binary operation on $P$,

- $a \odot b \leq c$ if and only if $a \leq b \rightarrow c$

for all $a, b, c \in P$. The last property is called adjointness.

Unfortunately, not every lattice $\left(L, \vee, \wedge,{ }^{\prime}\right)$ with an antitone involution ' can be converted into a residuated lattice $(L, \vee, \wedge, \odot, \rightarrow, 1)$ satisfying $x^{\prime}=x \rightarrow 0$ for all $x \in L$. For example, consider the non-modular lattice $\mathbf{N}_{5}=\left(N_{5}, \vee, \wedge\right)$ whose elements are $0, a, b, c, 1$ where 0 is the least and 1 the greatest element, $a<b$ and $c$ is incomparable with $a$ and $b$, see Figure 1:

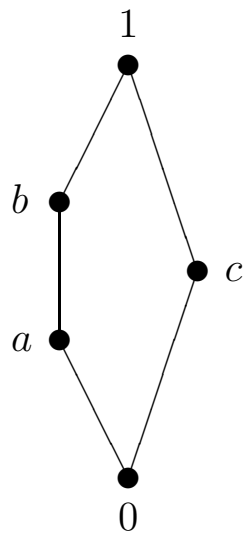

Fig. 1

It is easy to see that there exists exactly one antitone involution ' on $\left(N_{5}, \leq\right)$, namely $0^{\prime}=1, a^{\prime}=b, b^{\prime}=a, c^{\prime}=c$ and $1^{\prime}=0$. Suppose, $\mathbf{N}_{5}$ together with ' could be converted into a residuated poset $\left(N_{5}, \leq, \odot, \rightarrow, 1\right)$ satisfying $x^{\prime}=x \rightarrow 0$ for all $x \in N_{5}$. Since $x \odot y \leq x$ and $x \odot y \leq y$ for all $x, y \in N_{5}$ (see Theorem 2.17 in [1]) we have $c \odot a \leq 0$ which implies $c \leq a \rightarrow 0=a^{\prime}=b$, a contradiction. Thus $\mathbf{N}_{5}$ together with ' cannot be converted into a residuated poset $\left(N_{5}, \leq, \odot, \rightarrow, 1\right)$ satisfying $x^{\prime}=x \rightarrow 0$ for all $x \in N_{5}$.

Hence, it is a question whether such a lattice (or poset in general) can be extended to a residuated one by preserving the antitone involution. The aim of our paper is to show how such an extension can be constructed.

At first, we show that the unary operation $x^{\prime}:=x \rightarrow 0$ in a residuated lattice is antitone.

Lemma 1. Let $(P, \leq, \odot, \rightarrow, 1)$ be a residuated poset and put $x^{\prime}:=x \rightarrow 0$ for all $x \in P$ Then $x \leq x^{\prime \prime}$ for all $x \in P$ and' is antitone.

Proof. Let $a, b \in P$. Then the every of the following assertions implies the next one:

$$
\begin{aligned}
a \rightarrow 0 & \leq a \rightarrow 0, \\
(a \rightarrow 0) \odot a & \leq 0, \\
a \odot(a \rightarrow 0) & \leq 0, \\
a & \leq(a \rightarrow 0) \rightarrow 0, \\
a & \leq a^{\prime \prime} .
\end{aligned}
$$


Moreover, every of the following assertions implies the next one:

$$
\begin{aligned}
a & \leq b, \\
a & \leq(b \rightarrow 0) \rightarrow 0, \\
a \odot(b \rightarrow 0) & \leq 0, \\
(b \rightarrow 0) \odot a & \leq 0, \\
b \rightarrow 0 & \leq a \rightarrow 0, \\
b^{\prime} & \leq a^{\prime} .
\end{aligned}
$$

Our first extension result is as follows.

Theorem 2. Let $\mathbf{P}=\left(P, \leq,^{\prime}\right)$ be a poset with an antitone involution, assume $0=$ $c_{1}, \ldots, c_{4}=1 \notin P$, put $E(P):=P \cup\left\{c_{1}, \ldots, c_{4}\right\}$ and extend $\leq$ and ' from $P$ to $E(P)$ by $0<c_{2}<x<c_{3}<1$ for all $x \in P$ and $c_{i}^{\prime}:=c_{5-i}$ for $i=1, \ldots, 4$. Define binary operations $\odot$ and $\rightarrow$ on $E(P)$ as follows:

$$
\begin{aligned}
& 0 \odot x=x \odot 0:=0,1 \odot x=x \odot 1:=x, \\
& 0 \rightarrow x=x \rightarrow 1:=1, x \rightarrow 0:=x^{\prime}, 1 \rightarrow x:=x
\end{aligned}
$$

for $x \in E(P)$ and

$$
x \odot y:=\left\{\begin{array}{ll}
0 & \text { if } x \leq y^{\prime}, \\
c_{2} & \text { otherwise }
\end{array} \quad x \rightarrow y:= \begin{cases}1 & \text { if } x \leq y, \\
c_{3} & \text { otherwise }\end{cases}\right.
$$

for $x, y \in E(P) \backslash\{0,1\}$. Then $\mathbb{E}(\mathbf{P}):=(E(P), \leq, \odot, \rightarrow, 1)$ is a residuated poset with the antitone involution' satisfying $x^{\prime}=x \rightarrow 0$ for all $x \in E(P)$. (If $\mathbf{P}$ is already bounded then the least and greatest element of $\mathbf{P}$ may be identified with $c_{2}$ and $c_{3}$, respectively. If $\mathbf{P}$ has elements $a, b, c, d$ satisfying $a<b \leq x \leq c<d$ for all $x \in P \backslash\{a, d\}$ then $a, b, c, d$ may be identified with $c_{1}, \ldots, c_{4}$, respectively.)

Proof. Let $a, b, c \in E(P)$. Since $x^{\prime \prime}=x$ for all $x \in P$ and $c_{i}^{\prime \prime}=c_{5-i}^{\prime}=c_{5-(5-i)}=c_{i}$ for all $i=1, \ldots, 4,\left(E(P), \leq,^{\prime}, 0,1\right)$ is a bounded poset with an antitone involution.

If $\{a, b, c\} \cap\{0,1\} \neq \emptyset$ then, obviously, $(a \odot b) \odot c=a \odot(b \odot c)$. If $a, b, c \neq 0,1, a \leq b^{\prime}$ and $b \leq c^{\prime}$ then $(a \odot b) \odot c=0 \odot c=0=a \odot 0=a \odot(b \odot c)$.

If $a, b, c \neq 0,1, a \leq b^{\prime}$ and $b \not \leq c^{\prime}$ then $(a \odot b) \odot c=0 \odot c=0=a \odot c_{2}=a \odot(b \odot c)$.

If $a, b, c \neq 0,1, a \not \leq b^{\prime}$ and $b \leq c^{\prime}$ then $(a \odot b) \odot c=c_{2} \odot c=0=a \odot 0=a \odot(b \odot c)$.

If $a, b, c \neq 0,1, a \not \leq b^{\prime}$ and $b \not \leq c^{\prime}$ then $(a \odot b) \odot c=c_{2} \odot c=0=a \odot c_{2}=a \odot(b \odot c)$.

Therefore, $\odot$ is associative. Since $a \leq b^{\prime}$ is equivalent to $b \leq a^{\prime}, \odot$ is commutative.

If $a=0$ then $a \odot b=0$ and $a \leq b^{\prime}$.

If $b=0$ then $a \odot b=0$ and $a \leq b^{\prime}$.

If $a=1$ then $a \odot b=0$ and $a \leq b^{\prime}$ are equivalent to $b=0$.

If $b=1$ then $a \odot b=0$ and $a \leq b^{\prime}$ are equivalent to $a=0$.

If $a, b \neq 0,1$ then $a \odot b=0$ is equivalent to $a \leq b^{\prime}$.

Hence $a \odot b=0$ if and only if $a \leq b^{\prime}$.

If $a=0$ then $a \rightarrow b=1$ and $a \leq b$.

If $b=0$ then $a \rightarrow b=1$ and $a \leq b$ are equivalent to $a=0$.

If $a=1$ then $a \rightarrow b=1$ and $a \leq b$ are equivalent to $b=1$. 
If $b=1$ then $a \rightarrow b=1$ and $a \leq b$.

If $a, b \neq 0,1$ then $a \rightarrow b=1$ and $a \leq b$ are equivalent.

Hence $a \rightarrow b=1$ if and only if $a \leq b$.

If $a=0$ then $a \odot b \leq c$ and $a \leq b \rightarrow c$.

If $b=0$ then $a \odot b \leq c$ and $a \leq b \rightarrow c$.

If $c=0$ then $a \odot b \leq c$ and $a \leq b \rightarrow c$ are equivalent to $a \leq b^{\prime}$.

If $a=1$ then $a \odot b \leq c$ and $a \leq b \rightarrow c$ are equivalent to $b \leq c$.

If $b=1$ then $a \odot b \leq c$ and $a \leq b \rightarrow c$ are equivalent to $a \leq c$.

If $c=1$ then $a \odot b \leq c$ and $a \leq b \rightarrow c$.

If $a, b, c \neq 0,1$ then $a \odot b \leq c_{2}$ and $c_{3} \leq b \rightarrow c$ and hence $a \odot b \leq c$ and $a \leq b \rightarrow c$.

Thus the adjointness property holds.

As mentioned above, the non-modular lattice $\mathbf{N}_{5}$ with an antitone involution cannot be converted into a residuated lattice $\left(N_{5}, \vee, \wedge, \odot, \rightarrow, 1\right)$ with an antitone involution ' satisfying $x^{\prime}=x \rightarrow 0$ for all $x \in N_{5}$. Using Theorem 2 , we can extend $\mathbf{N}_{5}$ as follows.

Example 3. Theorem 2 applied to $\mathbf{N}_{5}$ yields the residuated lattice depicted in Figure 2:

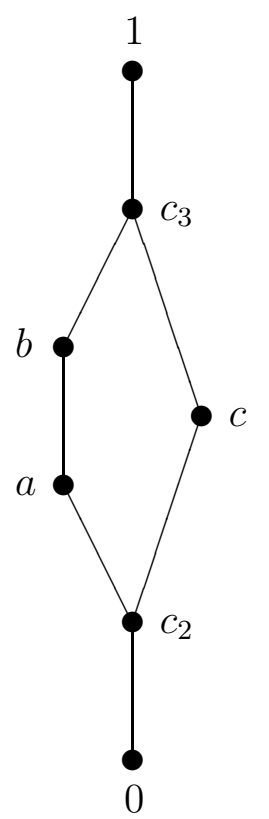

Fig. 2

with operation tables

\begin{tabular}{l|lllllll}
$\odot$ & 0 & $c_{2}$ & $a$ & $b$ & $c$ & $c_{3}$ & 1 \\
\hline 0 & 0 & 0 & 0 & 0 & 0 & 0 & 0 \\
$c_{2}$ & 0 & 0 & 0 & 0 & 0 & 0 & $c_{2}$ \\
$a$ & 0 & 0 & 0 & 0 & $c_{2}$ & $c_{2}$ & $a$ \\
$b$ & 0 & 0 & 0 & $c_{2}$ & $c_{2}$ & $c_{2}$ & $b$ \\
$c$ & 0 & 0 & $c_{2}$ & $c_{2}$ & 0 & $c_{2}$ & $c$ \\
$c_{3}$ & 0 & 0 & $c_{2}$ & $c_{2}$ & $c_{2}$ & $c_{2}$ & $c_{3}$ \\
1 & 0 & $c_{2}$ & $a$ & $b$ & $c$ & $c_{3}$ & 1
\end{tabular}

\begin{tabular}{l|lllllll}
$\rightarrow$ & 0 & $c_{2}$ & $a$ & $b$ & $c$ & $c_{3}$ & 1 \\
\hline 0 & 1 & 1 & 1 & 1 & 1 & 1 & 1 \\
$c_{2}$ & $c_{3}$ & 1 & 1 & 1 & 1 & 1 & 1 \\
$a$ & $b$ & $c_{3}$ & 1 & 1 & $c_{3}$ & 1 & 1 \\
$b$ & $a$ & $c_{3}$ & $c_{3}$ & 1 & $c_{3}$ & 1 & 1 \\
$c$ & $c$ & $c_{3}$ & $c_{3}$ & $c_{3}$ & 1 & 1 & 1 \\
$c_{3}$ & $c_{2}$ & $c_{3}$ & $c_{3}$ & $c_{3}$ & $c_{3}$ & 1 & 1 \\
1 & 0 & $c_{2}$ & $a$ & $b$ & $c$ & $c_{3}$ & 1
\end{tabular}

Observe there is only one possibility for the antitone involution. 
Let us note that if $\mathbf{P}=\left(P, \leq,^{\prime}\right)$ is a finite chain containing at least three elements (with unique antitone involution) then, using the construction from Theorem 2 , $\mathbf{P}$ can be converted into a residuated chain $\mathbb{E}(\mathbf{P})=(P, \leq, \odot, \rightarrow, 1)$ satisfying $x^{\prime}=x \rightarrow 0$ for all $x \in P$.

Corollary 4. If $(C, \leq)=\left(\left\{c_{1}, \ldots, c_{n}\right\}, \leq\right)$ is a finite chain $0=c_{1}<c_{2}<c_{3}<\cdots<$ $c_{n}=1$ with $n \geq 3$ elements,

$$
\begin{aligned}
& 0 \odot c_{i}=c_{i} \odot 0:=0,1 \odot c_{i}=c_{i} \odot 1:=c_{i}, \\
& 0 \rightarrow c_{i}=c_{i} \rightarrow 1:=1, c_{i} \rightarrow 0:=c_{n+1-i}, 1 \rightarrow c_{i}:=c_{i}
\end{aligned}
$$

for $i=1, \ldots, n$ and

$$
c_{i} \odot c_{j}:=\left\{\begin{array}{ll}
0 & \text { if } i+j \leq n+1, \\
c_{2} & \text { otherwise }
\end{array} \quad c_{i} \rightarrow c_{j}:= \begin{cases}1 & \text { if } i \leq j, \\
c_{n-1} & \text { otherwise }\end{cases}\right.
$$

for $i, j=2, \ldots, n-1$ then $(C, \leq, \odot, \rightarrow, 1)$ is a residuated lattice where $x^{\prime}:=x \rightarrow 0$ is an antitone involution.

Example 5. Theorem 2 or Corollary 4 applied to the five-element chain yields the residuated lattice depicted in Figure 3:

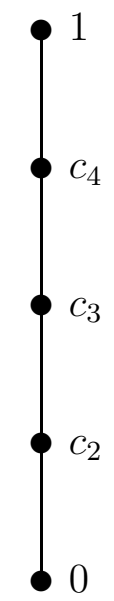

Fig. 3

with operation tables

\begin{tabular}{l|lllll}
$\odot$ & 0 & $c_{2}$ & $c_{3}$ & $c_{4}$ & 1 \\
\hline 0 & 0 & 0 & 0 & 0 & 0 \\
$c_{2}$ & 0 & 0 & 0 & 0 & $c_{2}$ \\
$c_{3}$ & 0 & 0 & 0 & $c_{2}$ & $c_{3}$ \\
$c_{4}$ & 0 & 0 & $c_{2}$ & $c_{2}$ & $c_{4}$ \\
1 & 0 & $c_{2}$ & $c_{3}$ & $c_{4}$ & 1
\end{tabular}

\begin{tabular}{l|lllll}
$\rightarrow$ & 0 & $c_{2}$ & $c_{3}$ & $c_{4}$ & 1 \\
\hline 0 & 1 & 1 & 1 & 1 & 1 \\
$c_{2}$ & $c_{4}$ & 1 & 1 & 1 & 1 \\
$c_{3}$ & $c_{3}$ & $c_{4}$ & 1 & 1 & 1 \\
$c_{4}$ & $c_{2}$ & $c_{4}$ & $c_{4}$ & 1 & 1 \\
1 & 0 & $c_{2}$ & $c_{3}$ & $c_{4}$ & 1
\end{tabular}

Here $c_{3}$ of Theorem 2 corresponds to $c_{4}$ of Example 5 .

If the poset $\mathbf{P}$ in question is a lattice, we can also apply the construction of ${ }^{\prime}, \odot$ and $\rightarrow$ from Theorem 2 to obtain a residuated lattice $\mathbb{E}(\mathbf{P})$. Hence, we can state the following.

Corollary 6. Let $\mathbf{L}=\left(L, \vee, \wedge,^{\prime}\right)$ be a lattice with an antitone involution'. Then $\mathbf{L}$ can be extended to a residuated lattice $\mathbb{E}(\mathbf{L})$ with an antitone involution where the operations ', $\odot$ and $\rightarrow$ are constructed as in Theorem 2 and ' coincides in $L$ with the original one. 
Recall from [2] and [11] that a lattice $\mathbf{L}=\left(L, \vee, \wedge,{ }^{\prime}\right)$ with an antitone involution is called a pseudo-Kleene algebra if it satisfies the identities

(1) $x \wedge x^{\prime} \leq y \vee y^{\prime}$,

(2) $x \wedge\left(x^{\prime} \vee y\right) \approx\left(x \wedge x^{\prime}\right) \vee(x \wedge y)$.

$\mathbf{L}$ is called a Kleene algebra if it is a distributive pseudo-Kleene algebra. In this case (2) can be omitted since it follows by distributivity. Kleene algebras and pseudo-Kleene algebras are considered as an algebraic axiomatization of a propositional logic satisfying De Morgan's laws and the double negation law, but not necessarily the law of excluded middle because the antitone involution' need not be a complementation, see also [9]. An example of a Kleene algebra is depicted in Figure 4:

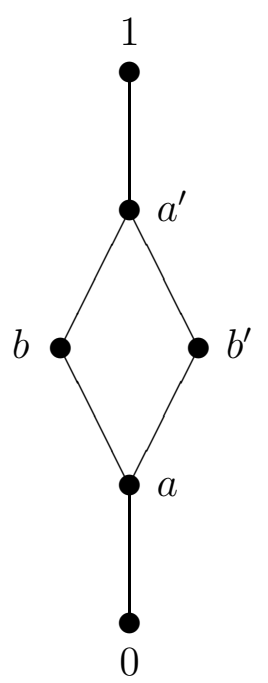

Fig. 4

A pseudo-Kleene algebra which is not a Kleene algebra is visualized in Figure 5:

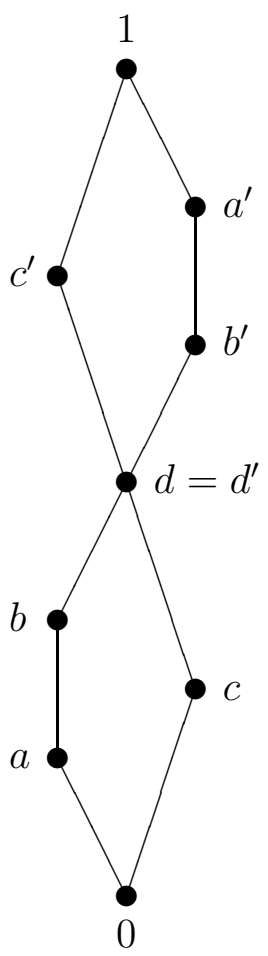

Fig. 5 
One can easily check that if $\mathbf{L}$ satisfies (1) and (2) then so does $\mathbb{E}(\mathbf{L})$ and if $\mathbf{L}$ is distributive then also $\mathbb{E}(\mathbf{L})$ has this property, too. Hence, we can state

Corollary 7. Every pseudo-Kleene algebra or every Kleene algebra can be extended to a residuated pseudo-Kleene algebra or a residuated Kleene-algebra, respectively.

Hence, also the logic axiomatized by Kleene algebras or pseudo-Kleene algebras can be extended to a kind of fuzzy logics.

Motivated by the previous, we can extend every poset with an antitone involution to a residuated poset by means of a finite chain with an even number of at least four elements. The precise formulation is as follows:

Theorem 8. Let $\left(P, \leq,^{\prime}\right)$ be a poset with an antitone involution, $n$ an integer $>1$, $0=c_{1}, \ldots, c_{2 n}=1 \notin P$ and $Q:=P \cup\left\{c_{1}, \ldots, c_{2 n}\right\}$. Extend $\leq$ and' from $P$ to $Q$ by

$$
c_{1}<\cdots<c_{n}<x<c_{n+1}<\cdots<c_{2 n}
$$

for $x \in P$ and put $c_{i}^{\prime}:=c_{2 n+1-i}$ for $i=1, \ldots, 2 n$. Define binary operations $\odot$ and $\rightarrow$ on $Q$ as follows:

$$
\begin{aligned}
& 0 \odot x=x \odot 0:=0,1 \odot x=x \odot 1:=x, \\
& 0 \rightarrow x=x \rightarrow 1:=1, x \rightarrow 0:=x^{\prime}, 1 \rightarrow x:=x
\end{aligned}
$$

for $x \in Q$ and

$$
\begin{gathered}
x \odot y:=\left\{\begin{array}{ll}
0 & \text { if } x \leq y^{\prime}, \\
c_{2} & \text { otherwise }
\end{array} \quad x \rightarrow y:= \begin{cases}1 & \text { if } x \leq y, \\
c_{2 n-1} & \text { otherwise }\end{cases} \right. \\
c_{i} \odot c_{j}:=\left\{\begin{array}{ll}
0 & \text { if } i+j \leq 2 n+1, \\
c_{2} & \text { otherwise }
\end{array} \quad c_{i} \rightarrow c_{j}:= \begin{cases}1 & \text { if } i \leq j, \\
c_{2 n-1} & \text { otherwise }\end{cases} \right. \\
c_{i} \odot x=x \odot c_{i}:=c_{i} \odot c_{n+1}, c_{i} \rightarrow x:=c_{i} \rightarrow c_{n}, x \rightarrow c_{i}:=c_{n+1} \rightarrow c_{i}
\end{gathered}
$$

for $x, y \in P$ and $i, j=2, \ldots, 2 n-1$. Then $(Q, \leq, \odot, \rightarrow, 1)$ is a residuated poset with the antitone involution' satisfying $x^{\prime}=x \rightarrow 0$ for all $x \in Q$.

Proof. We apply Theorem 2 to the poset $\left(P \cup\left\{c_{3}, \ldots, c_{2 n-2}\right\}, \leq,^{\prime}\right)$ with the antitone involution '. The element $c_{3}$ of Theorem 2 corresponds to the element $c_{2 n-1}$ of Theorem 8 . According to Theorem 2 we have for all $x \in Q$

$$
\begin{aligned}
& 0 \odot x=x \odot 0=0,1 \odot x=x \odot 1=x, \\
& 0 \rightarrow x=x \rightarrow 1=1, x \rightarrow 0=x^{\prime}, 1 \rightarrow x=x .
\end{aligned}
$$

Moreover, we have for all $x, y \in P$ and $i, j \in\{2, \ldots, 2 n-1\}$

$$
\begin{aligned}
& x \odot y=\left\{\begin{array}{ll}
0 & \text { if } x \leq y^{\prime}, \\
c_{2} & \text { otherwise }
\end{array} \quad x \rightarrow y= \begin{cases}1 & \text { if } x \leq y, \\
c_{2 n-1} & \text { otherwise }\end{cases} \right. \\
& c_{i} \odot c_{j}=\left\{\begin{array}{ll}
0 & \text { if } i \leq 2 n+1-j, \\
c_{2} & \text { otherwise }
\end{array} \quad c_{i} \rightarrow c_{j}= \begin{cases}1 & \text { if } i \leq j, \\
c_{2 n-1} & \text { otherwise }\end{cases} \right.
\end{aligned}
$$




$$
\begin{gathered}
c_{i} \odot x=x \odot c_{i}= \begin{cases}0 & \text { if } i \leq n, \\
c_{2} & \text { otherwise }\end{cases} \\
c_{i} \rightarrow x=\left\{\begin{array}{ll}
1 & \text { if } i \leq n, \\
c_{2 n-1} & \text { otherwise }
\end{array} \quad x \rightarrow c_{i}= \begin{cases}1 & \text { if } i>n, \\
c_{2 n-1} & \text { otherwise }\end{cases} \right.
\end{gathered}
$$

The poset $(Q, \leq)$ of Theorem 8 is visualized in Figure 6 :

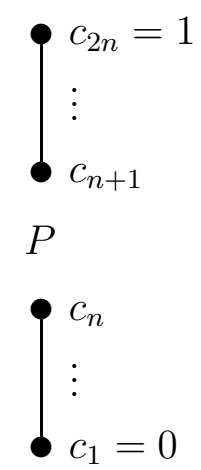

Fig. 6

Let $P$ be a set and $\mathbf{P}_{1}=(P \times\{1\}, \leq)$ and $\mathbf{P}_{2}=(P \times\{2\}, \leq)$ posets. We call $\mathbf{P}_{2}$ the dual of $\mathbf{P}_{1}$ if for all $x, y \in P$ we have $(x, 2) \leq(y, 2)$ if and only if $(y, 1) \leq(x, 1)$. Similarly as before, a poset together with its dual can be extended to a residuated poset by means of a finite chain with at least four elements.

Theorem 9. Let $(P \times\{1\}, \leq)$ be a poset, $(P \times\{2\}, \leq)$ denote its dual, $n$ an integer $>1$, $k$ a non-negative integer, $0=c_{1}, \ldots, c_{2 n+k}=1 \notin P \times\{1,2\}$ and $Q:=(P \times\{1,2\}) \cup$ $\left\{c_{1}, \ldots, c_{2 n+k}\right\}$. Extend $\leq$ to $Q$ by

$$
c_{1}<\cdots<c_{n}<(x, 1)<c_{n+1}<\cdots<c_{n+k}<(y, 2)<c_{n+k+1}<\cdots<c_{2 n+k}
$$

for $x, y \in P$ and put $c_{i}^{\prime}:=c_{2 n+k+1-i}$ for $i=1, \ldots, 2 n+k$ and $(x, i)^{\prime}:=(x, 3-i)$ for $x \in P$ and $i \in\{1,2\}$. Define binary operations $\odot$ and $\rightarrow$ on $Q$ as follows:

$$
\begin{aligned}
& 0 \odot x=x \odot 0:=0,1 \odot x=x \odot 1:=x, \\
& 0 \rightarrow x=x \rightarrow 1:=1, x \rightarrow 0:=x^{\prime}, 1 \rightarrow x:=x
\end{aligned}
$$

for $x \in Q$,

$$
\begin{aligned}
& (x, i) \odot(y, j):= \begin{cases}0 \quad & \text { if } i=j=1 \text { or }((i, j)=(1,2) \text { and } x \leq y) \text { or } \\
& (i, j)=(2,1) \text { and } y \leq x, \\
c_{2} \quad \text { otherwise }\end{cases} \\
& (x, i) \rightarrow(y, j):= \begin{cases}1 & \text { if }(i=j=1 \text { and } x \leq y) \text { or }(i, j)=(1,2) \text { or } \\
& (i=j=2 \text { and } y \leq x), \\
c_{2 n+k-1} \quad \text { otherwise }\end{cases}
\end{aligned}
$$

for $x, y \in P$ and $i, j \in\{1,2\}$ and

$$
c_{i} \odot c_{j}:=\left\{\begin{array}{ll}
0 & \text { if } i+j \leq 2 n+k+1, \\
c_{2} & \text { otherwise }
\end{array} \quad c_{i} \rightarrow c_{j}:= \begin{cases}1 & \text { if } i \leq j, \\
c_{2 n+k-1} & \text { otherwise }\end{cases}\right.
$$




$$
\begin{aligned}
& c_{i} \odot(x, 1)=(x, 1) \odot c_{i}:=c_{i} \odot c_{n+1}, c_{i} \rightarrow(x, 1):=c_{i} \rightarrow c_{n},(x, 1) \rightarrow c_{i}:=c_{n+1} \rightarrow c_{i}, \\
& c_{i} \odot(x, 2)=(x, 2) \odot c_{i}:=c_{i} \odot c_{n+k+1}, c_{i} \rightarrow(x, 2):=c_{i} \rightarrow c_{n+k}, \\
& (x, 2) \rightarrow c_{i}:=c_{n+k+1} \rightarrow c_{i}
\end{aligned}
$$

for $i, j \in\{2, \ldots, 2 n+k-1\}$ and $x \in P$. Then $(Q, \leq, \odot, \rightarrow, 1)$ is a residuated poset with an antitone involution' satisfying $x^{\prime}=x \rightarrow 0$ for all $x \in Q$.

Proof. We apply Theorem 2 to the poset $\left(P \cup\left\{c_{3}, \ldots, c_{2 n+k-2}\right\}, \leq,^{\prime}\right)$ with the antitone involution '. The element $c_{3}$ of Theorem 2 corresponds to the element $c_{2 n+k-1}$ of Theorem 9 . According to Theorem 2 we have for all $x \in Q$

$$
\begin{aligned}
& 0 \odot x=x \odot 0=0,1 \odot x=x \odot 1=x, \\
& 0 \rightarrow x=x \rightarrow 1=1, x \rightarrow 0=x^{\prime}, 1 \rightarrow x=x .
\end{aligned}
$$

Moreover, we have for all $x, y \in P$ and $i, j \in\{1,2\}$

$$
\begin{aligned}
(x, i) \odot(y, j) & = \begin{cases}0 & \text { if }(x, i) \leq(y, 3-j), \\
c_{2} & \text { otherwise }\end{cases} \\
(x, i) \rightarrow(y, j) & = \begin{cases}1 & \text { if }(x, i) \leq(y, j), \\
c_{2 n+k-1} & \text { otherwise }\end{cases}
\end{aligned}
$$

Further, we have for all $i, j \in\{2, \ldots, 2 n+k-1\}$

$$
c_{i} \odot c_{j}=\left\{\begin{array}{ll}
0 & \text { if } i \leq 2 n+k+1-j, \\
c_{2} & \text { otherwise }
\end{array} c_{i} \rightarrow c_{j}= \begin{cases}1 & \text { if } i \leq j, \\
c_{2 n+k-1} & \text { otherwise }\end{cases}\right.
$$

Finally, we have for all $i \in\{2, \ldots, 2 n+k-1\}$ and $j \in\{1,2\}$

$$
\begin{gathered}
c_{i} \odot(x, j)=(x, j) \odot c_{i}= \begin{cases}0 & \text { if } c_{i} \leq(x, 3-j), \\
c_{2} & \text { otherwise }\end{cases} \\
c_{i} \rightarrow(x, j)=\left\{\begin{array}{ll}
1 & \text { if } c_{i} \leq(x, j), \\
c_{2 n+k-1} & \text { otherwise }
\end{array} \quad(x, j) \rightarrow c_{i}= \begin{cases}1 & \text { if }(x, j) \leq c_{i}, \\
c_{2 n+k-1} & \text { otherwise }\end{cases} \right.
\end{gathered}
$$

The poset $(Q, \leq)$ of Theorem 8 is visualized in Figure 7 :

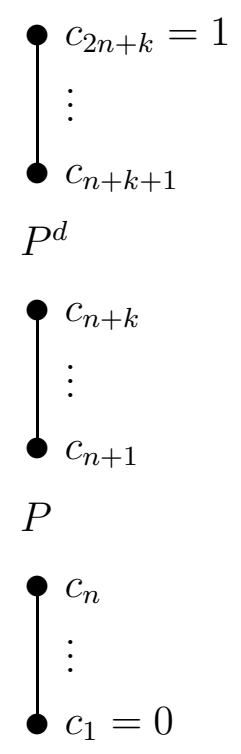

Fig. 7 
It is obvious that the constructions described in Theorems 8 and 9 can be generalized to finitely many posets and finite chains in between them. For instance, if we have posets $P_{1}, P_{2}, P_{3}$, their duals $P_{1}^{d}, P_{2}^{d}, P_{3}^{d}$ and a poset $P$ with an antitone involution then we can construct, similarly as before, a poset with an antitone involution of the form

$$
\cdots<x_{1}<\cdots<x_{2}<\cdots<x_{3}<\cdots<x<\cdots<y_{3}<\cdots<y_{2}<\cdots<y_{1}<\cdots
$$

for all $x_{1} \in P_{1}, x_{2} \in P_{2}, x_{3} \in P_{3}, x \in P, y_{3} \in P_{3}^{d}, y_{2} \in P_{2}^{d}, y_{1} \in P_{1}^{d}$ where the dots (from left to right) indicate finite chains with $r, s, t, u, u, t, s, r$ elements, respectively, and then we can apply Theorem 2 to this poset with antitone involution.

The following result is well known, see e.g. [1] or [12.

Lemma 10. Let $\left(B, \vee, \wedge,{ }^{\prime}, p, q\right)$ be a Boolean algebra and put

$$
\begin{aligned}
x \odot y & :=x \wedge y, \\
x \rightarrow y & :=x^{\prime} \vee y
\end{aligned}
$$

for every $x, y \in B$. Then $(B, \leq, \odot, \rightarrow, q)$ is a residuated poset.

In the following we extend a Boolean algebra $\left(B, \vee, \wedge,{ }^{\prime}, p, q\right)$ to a residuated lattice $(Q, \vee, \wedge, \odot, \rightarrow, 1)$ with an antitone involution $x^{\prime}=x \rightarrow 0$ by means of a (finite) chain such that the operations $\odot$ and $\rightarrow$ coincide on $B$ with those mentioned in Lemma 10 .

Theorem 11. Let $\left(B, \vee, \wedge,{ }^{\prime}, p, q\right)$ be a Boolean algebra, $n$ a positive integer, $0=c_{1}, \ldots$ $\ldots, c_{2 n}=1 \notin B$ and $Q:=B \cup\left\{c_{1}, \ldots, c_{2 n}\right\}$. Extend $\leq$ and' from $B$ to $Q$ by

$$
c_{1}<\cdots<c_{n}<x<c_{n+1}<\cdots<c_{2 n}
$$

for $x \in B$ and put $c_{i}^{\prime}:=c_{2 n+1-i}$ for $i=1, \ldots, 2 n$. Define binary operations $\odot$ and $\rightarrow$ on $Q$ as follows:

$$
x \odot y:=\left\{\begin{array}{ll}
0 & \text { if } x \leq y^{\prime}, \\
x \wedge y & \text { otherwise }
\end{array} \quad x \rightarrow y:= \begin{cases}1 & \text { if } x \leq y, \\
x^{\prime} \vee y & \text { otherwise }\end{cases}\right.
$$

for $x, y \in Q$. Then $(Q, \leq, \odot, \rightarrow, 1)$ is a residuated lattice with an antitone involution $x^{\prime}=x \rightarrow 0$ where for all $x, y \in B$ we have

$$
\begin{aligned}
x \odot y & =x \wedge y, \\
x \rightarrow y & =x^{\prime} \vee y .
\end{aligned}
$$

Proof. Let $a, b, c \in Q, d, e \in B$ and $i, j, k \in\{1, \ldots, 2 n\}$. Within this proof

$$
\begin{aligned}
& a<B \text { means } a<x \text { for all } x \in B, \\
& a>B \text { means } a>x \text { for all } x \in B, \\
& i \vee j:=\max (i, j) \\
& i \wedge j:=\min (i, j) .
\end{aligned}
$$


Obviously, $\left(Q, \leq{ }^{\prime}, 0,1\right)$ is a bounded lattice with an antitone involution. We have

$$
\begin{aligned}
& a \odot b=b \odot a \text { since } a \leq b^{\prime} \text { is equivalent to } b \leq a^{\prime}, \\
& 0 \odot a=0, \\
& 1 \odot a= \begin{cases}0=a & \text { if } a=0, \\
1 \wedge a=a & \text { otherwise }\end{cases} \\
& 0 \rightarrow a=a \rightarrow 1=1, \\
& a \rightarrow 0= \begin{cases}1=0^{\prime}=a^{\prime} & \text { if } a=0, \\
a^{\prime} \vee 0=a^{\prime} & \text { otherwise }\end{cases} \\
& d \odot e= \begin{cases}c_{1}=d \wedge e & \text { if } d \leq e^{\prime}, \\
d \wedge e & \text { otherwise }\end{cases} \\
& d \rightarrow e= \begin{cases}1=d^{\prime} \vee e & \text { if } d \leq e, \\
d^{\prime} \vee e & \text { otherwise. }\end{cases}
\end{aligned}
$$

This shows

$$
\begin{aligned}
& 0 \odot x=x \odot 0=0,1 \odot x=x \odot 1=x, \\
& 0 \rightarrow x=x \rightarrow 1=1, x \rightarrow 0=x^{\prime}, 1 \rightarrow x=x
\end{aligned}
$$

$(x \in Q)$ and

$$
\begin{aligned}
x \odot y & =x \wedge y, \\
x \rightarrow y & =x^{\prime} \vee y
\end{aligned}
$$

for all $x, y \in B$.

If $a \leq b^{\prime}$ and $b \leq c^{\prime}$ then $(a \odot b) \odot c=0 \odot c=0=a \odot 0=a \odot(b \odot c)$.

If $a \leq b^{\prime}$ and $b \not \leq c^{\prime}$ then $(a \odot b) \odot c=0 \odot c=0=a \odot(b \wedge c)=a \odot(b \odot c)$.

If $a \not \leq b^{\prime}$ and $b \leq c^{\prime}$ then $(a \odot b) \odot c=(a \wedge b) \odot c=0=a \odot 0=a \odot(b \odot c)$.

Now consider the case $a \not \leq b^{\prime}$ and $b \not \leq c^{\prime}$. Then

$$
\begin{aligned}
& (a \odot b) \odot c=(a \wedge b) \odot c, \\
& a \odot(b \odot c)=a \odot(b \wedge c) .
\end{aligned}
$$

If $a, b, c \in B$ then $(a \wedge b) \odot c=(a \wedge b) \wedge c=a \wedge(b \wedge c)=a \odot(b \wedge c)$.

If $a, b \in B$ and $c \notin B$ then $c>B$ and hence $(a \wedge b) \odot c=(a \wedge b) \wedge c=a \wedge b=a \odot b=a \odot(b \wedge c)$. If $a \in B, b \notin B$ and $c \in B$ then $b>B$ and hence $(a \wedge b) \odot c=a \odot c=a \odot(b \wedge c)$.

If $a \in B$ and $b, c \notin B$ then $b>B$ and $\left(a \leq c^{\prime}\right.$ if and only if $\left.a \leq b^{\prime} \vee c^{\prime}\right)$. Hence $(a \wedge b) \odot c=a \odot c=0=a \odot(b \wedge c)$ if $a \leq c^{\prime}$ and $(a \wedge b) \odot c=a \odot c=a \wedge c=(a \wedge b) \wedge c=$ $a \wedge(b \wedge c)=a \odot(b \wedge c)$ otherwise.

If $a \notin B$ and $b, c \in B$ then $a>B$ and hence $(a \wedge b) \odot c=b \odot c=b \wedge c=(a \wedge b) \wedge c=$ $a \wedge(b \wedge c)=a \odot(b \wedge c)$.

If $a \notin B, b \in B$ and $c \notin B$ then $a, c>B$ and hence $(a \wedge b) \odot c=b \odot c=b \wedge c=b=$ $a \wedge b=a \odot b=a \odot(b \wedge c)$.

If $a, b \notin B$ and $c \in B$ then $b>B$ and $\left(a \leq c^{\prime}\right.$ if and only if $\left.a \wedge b \leq c^{\prime}\right)$. Hence $(a \wedge b) \odot c=0=a \odot c=a \odot(b \wedge c)$ if $a \leq c^{\prime}$ and $(a \wedge b) \odot c=(a \wedge b) \wedge c=a \wedge(b \wedge c)=$ $a \wedge c=a \odot c=a \odot(b \wedge c)$ otherwise.

Finally, assume $a, b, c \notin B$. Without loss of generality, $(a, b, c)=\left(c_{i}, c_{j}, c_{k}\right)$. Now we have

$$
c_{i} \odot c_{j}= \begin{cases}0 & \text { if } i+j \leq 2 n+1 \\ c_{i \wedge j} & \text { otherwise }\end{cases}
$$


If $i+j, j+k \leq 2 n+1$ then $\left(c_{i} \odot c_{j}\right) \odot c_{k}=0 \odot c_{k}=0=c_{i} \odot 0=c_{i} \odot\left(c_{j} \odot c_{k}\right)$.

If $i+j \leq 2 n+1<j+k$ then $i+(j \wedge k)=(i+j) \wedge(i+k) \leq i+j \leq 2 n+1$ and hence $\left(c_{i} \odot c_{j}\right) \odot c_{k}=0 \odot c_{k}=0=c_{i} \odot c_{j \wedge k}=c_{i} \odot\left(c_{j} \odot c_{k}\right)$.

If $j+k \leq 2 n+1<i+j$ then $(i \wedge j)+k=(i+k) \wedge(j+k) \leq j+k \leq 2 n+1$ and hence $\left(c_{i} \odot c_{j}\right) \odot c_{k}=c_{i \wedge j} \odot c_{k}=0=c_{i} \odot 0=c_{i} \odot\left(c_{j} \odot c_{k}\right)$.

If $i+j, j+k>2 n+1$ then

$$
\begin{gathered}
(i \wedge j)+k=(i+k) \wedge(j+k) \leq 2 n+1 \text { if and only if } i+k \leq 2 n+1 \\
i+(j \wedge k)=(i+j) \wedge(i+k) \leq 2 n+1 \text { if and only if } i+k \leq 2 n+1
\end{gathered}
$$

and hence $\left(c_{i} \odot c_{j}\right) \odot c_{k}=c_{i \wedge j} \odot c_{k}=c_{i} \odot c_{j \wedge k}=c_{i} \odot\left(c_{j} \odot c_{k}\right)$.

This shows that $\odot$ is associative. Since $a \leq b^{\prime}$ is equivalent to $b \leq a^{\prime}, \odot$ is commutative. Therefore, $(Q, \odot, 1)$ is a commutative monoid.

If $a \leq b^{\prime}$ and $b \leq c$ then $a \odot b=0 \leq c$ and $a \leq 1=b \rightarrow c$.

If $a \leq b^{\prime}$ and $b \not \leq c$ then $a \odot b=0 \leq c$, and $a \leq b \rightarrow c$ since $a \leq b^{\prime} \vee c$.

If $a \not \leq b^{\prime}$ and $b \leq c$ then $a \odot b \leq c$ since $a \wedge b \leq c$, and $a \leq 1=b \rightarrow c$.

Now assume $a \not \leq b^{\prime}$ and $b \not \leq c$.

If $a, b, c \in B$ then $a \wedge b \leq c$ if and only if $a \leq b^{\prime} \vee c$ because of Lemma 10.

If $a, b \in B$ and $c \notin B$ then $c<B$ and hence $a \wedge b \not \leq c$ and $a \not \leq b^{\prime}=b^{\prime} \vee c$.

If $b \notin B$ and $c \in B$ then $b>B$ and hence $a \wedge b \leq c$ and $a \leq b^{\prime} \vee c$ are both equivalent to $a \leq c$.

If $a \in B$ and $b, c \notin B$ then $b>B$ and hence $a \wedge b \leq c$ and $a \leq b^{\prime} \vee c$ are both equivalent to $a \leq c$.

If $a \notin B$ and $b, c \in B$ then $a>B$ and hence $a \wedge b=b \not \leq c$ and $a \not \leq b b^{\prime} \vee c$.

If $a \notin B, b \in B$ and $c \notin B$ then $c<B<a$ and hence $a \wedge b=b \not \leq c$ and $a \not \leq b^{\prime}=b^{\prime} \vee c$.

Finally, assume $a, b, c \notin B$. Without loss of generality, $(a, b, c)=\left(c_{i}, c_{j}, c_{k}\right)$. Now we have

$$
c_{i} \rightarrow c_{j}= \begin{cases}1 & \text { if } i \leq j, \\ c_{(2 n+1-i) \vee j} & \text { otherwise }\end{cases}
$$

If $i+j \leq 2 n+1$ and $j \leq k$ then $c_{i} \odot c_{j}=0 \leq c_{k}$ and $c_{i} \leq 1=c_{j} \rightarrow c_{k}$.

If $i+j \leq 2 n+1$ and $j>k$ then $c_{i} \odot c_{j}=0 \leq c_{k}$ and $c_{i} \leq c_{(2 n+1-j) \vee k}$ since $i \leq 2 n+1-j$.

If $i+j>2 n+1$ and $j \leq k$ then $c_{i} \odot c_{j}=c_{i \wedge j} \leq c_{k}$ and $c_{i} \leq c_{2 n}=c_{j} \rightarrow c_{k}$.

Finally, assume $i+j>2 n+1$ and $j>k$. Then the following are equivalent:

$$
\begin{aligned}
c_{i} \odot c_{j} & \leq c_{k}, \\
c_{i \wedge j} & \leq c_{k}, \\
i \wedge j & \leq k, \\
i & \leq k .
\end{aligned}
$$

Moreover, the following are equivalent:

$$
\begin{aligned}
c_{i} & \leq c_{j} \rightarrow c_{k}, \\
c_{i} & \leq c_{(2 n+1-j) \vee k} \\
i & \leq(2 n+1-j) \vee k, \\
i+j & \leq(2 n+1) \vee(k+j), \\
i+j & \leq k+j, \\
i & \leq k
\end{aligned}
$$

This proves adjointness. 
The poset $(Q, \leq)$ of Theorem 8 is visualized in Figure 8:

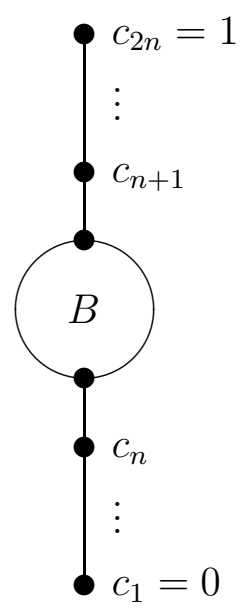

Fig. 8

Example 12. We consider the special case where $\left(B, \vee, \wedge,{ }^{\prime}, p, q\right)$ is the eight-element Boolean algebra and $n=2$. The poset $(Q, \leq)$ is visualized in Figure 9:

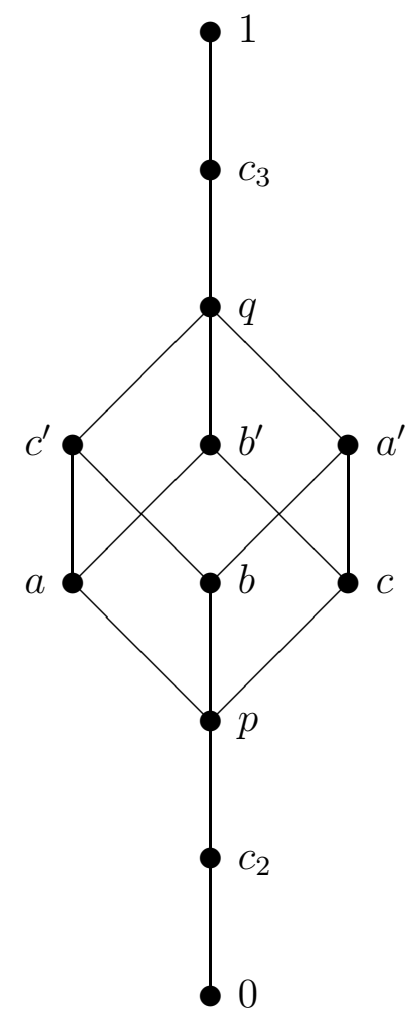

Fig. 9 
The corresponding residuated lattice has the operations

\begin{tabular}{l|llllllllllll}
$\odot$ & 0 & $c_{2}$ & $p$ & $a$ & $b$ & $c$ & $a^{\prime}$ & $b^{\prime}$ & $c^{\prime}$ & $q$ & $c_{3}$ & 1 \\
\hline 0 & 0 & 0 & 0 & 0 & 0 & 0 & 0 & 0 & 0 & 0 & 0 & 0 \\
$c_{2}$ & 0 & 0 & 0 & 0 & 0 & 0 & 0 & 0 & 0 & 0 & 0 & $c_{2}$ \\
$p$ & 0 & 0 & 0 & 0 & 0 & 0 & 0 & 0 & 0 & 0 & $p$ & $p$ \\
$a$ & 0 & 0 & 0 & $a$ & 0 & 0 & 0 & $a$ & $a$ & $a$ & $a$ & $a$ \\
$b$ & 0 & 0 & 0 & 0 & $b$ & 0 & $b$ & 0 & $b$ & $b$ & $b$ & $b$ \\
$c$ & 0 & 0 & 0 & 0 & 0 & $c$ & $c$ & $c$ & 0 & $c$ & $c$ & $c$ \\
$a^{\prime}$ & 0 & 0 & 0 & 0 & $b$ & $c$ & $a^{\prime}$ & $c$ & $b$ & $a^{\prime}$ & $a^{\prime}$ & $a^{\prime}$ \\
$b^{\prime}$ & 0 & 0 & 0 & $a$ & 0 & $c$ & $c$ & $b^{\prime}$ & $a$ & $b^{\prime}$ & $b^{\prime}$ & $b^{\prime}$ \\
$c^{\prime}$ & 0 & 0 & 0 & $a$ & $b$ & 0 & $b$ & $a$ & $c^{\prime}$ & $c^{\prime}$ & $c^{\prime}$ & $c^{\prime}$ \\
$q$ & 0 & 0 & 0 & $a$ & $b$ & $c$ & $a^{\prime}$ & $b^{\prime}$ & $c^{\prime}$ & $q$ & $q$ & $q$ \\
$c_{3}$ & 0 & 0 & $p$ & $a$ & $b$ & $c$ & $a^{\prime}$ & $b^{\prime}$ & $c^{\prime}$ & $q$ & $c_{3}$ & $c_{3}$ \\
1 & 0 & $c_{2}$ & $p$ & $a$ & $b$ & $c$ & $a^{\prime}$ & $b^{\prime}$ & $c^{\prime}$ & $q$ & $c_{3}$ & 1 \\
$\rightarrow$ & & & & & & & & & & & \\
$\rightarrow$ & 0 & $c_{2}$ & $p$ & $a$ & $b$ & $c$ & $a^{\prime}$ & $b^{\prime}$ & $c^{\prime}$ & $q$ & $c_{3}$ & 1 \\
\hline 0 & 1 & 1 & 1 & 1 & 1 & 1 & 1 & 1 & 1 & 1 & 1 & 1 \\
$c_{2}$ & $c_{3}$ & 1 & 1 & 1 & 1 & 1 & 1 & 1 & 1 & 1 & 1 & 1 \\
$p$ & $q$ & $q$ & 1 & 1 & 1 & 1 & 1 & 1 & 1 & 1 & 1 & 1 \\
$a$ & $a^{\prime}$ & $a^{\prime}$ & $a^{\prime}$ & 1 & $a^{\prime}$ & $a^{\prime}$ & $a^{\prime}$ & 1 & 1 & 1 & 1 & 1 \\
$b$ & $b^{\prime}$ & $b^{\prime}$ & $b^{\prime}$ & $b^{\prime}$ & 1 & $b^{\prime}$ & 1 & $b^{\prime}$ & 1 & 1 & 1 & 1 \\
$c$ & $c^{\prime}$ & $c^{\prime}$ & $c^{\prime}$ & $c^{\prime}$ & $c^{\prime}$ & 1 & 1 & 1 & $c^{\prime}$ & 1 & 1 & 1 \\
$a^{\prime}$ & $a$ & $a$ & $a$ & $a$ & $c^{\prime}$ & $b^{\prime}$ & 1 & $b^{\prime}$ & $c^{\prime}$ & 1 & 1 & 1 \\
$b^{\prime}$ & $b$ & $b$ & $b$ & $c^{\prime}$ & $b$ & $a^{\prime}$ & $a^{\prime}$ & 1 & $c^{\prime}$ & 1 & 1 & 1 \\
$c^{\prime}$ & $c$ & $c$ & $c$ & $b^{\prime}$ & $a^{\prime}$ & $c$ & $a^{\prime}$ & $b^{\prime}$ & 1 & 1 & 1 & 1 \\
$q$ & $p$ & $p$ & $p$ & $a$ & $b$ & $c$ & $a^{\prime}$ & $b^{\prime}$ & $c^{\prime}$ & 1 & 1 & 1 \\
$c_{3}$ & $c_{2}$ & $c_{2}$ & $p$ & $a$ & $b$ & $c$ & $a^{\prime}$ & $b^{\prime}$ & $c^{\prime}$ & $q$ & 1 & 1 \\
1 & 0 & $c_{2}$ & $p$ & $a$ & $b$ & $c$ & $a^{\prime}$ & $b^{\prime}$ & $c^{\prime}$ & $q$ & $c_{3}$ & 1
\end{tabular}

\section{References}

[1] R. Bělohlávek, Fuzzy Relational Systems. Foundations and Principles. Springer, New York 2002. ISBN 978-1-4613-5168-9.

[2] I. Chajda, A note on pseudo-Kleene algebras. Acta Univ. Palack. Olomuc. Fac. Rerum Natur. Math. 55 (2016), 39-45.

[3] I. Chajda, A representation of residuated lattices satisfying the double negation law. Soft Computing 22 (2018), 1773-1776.

[4] I. Chajda and D. Fazio, On residuation in paraorthomodular lattices. Soft Computing. DOI 10.1007/s00500-020-04699-w .

[5] I. Chajda and H. Länger, Residuated operators in complemented posets. Asian-Eur. J. Math. 11 (2018), 1850097, 15 pp. 
[6] I. Chajda and H. Länger, Residuated structures derived from commutative idempotent semirings. Discuss. Math. Gen. Algebra Appl. 39 (2019), 23-33.

[7] I. Chajda and H. Länger, Residuation in modular lattices and posets. Asian-Eur. J. Math. 12 (2019), 1950092, 10 pp.

[8] L. C. Ciungu, Non-commutative Multiple-Valued Logic Algebras. Springer, Cham 2014. ISBN 978-3-319-01588-0.

[9] P. Jipsen, From semirings to residuated Kleene lattices. Studia Logica 76 (2004), 291-303.

[10] P. Jipsen and C. Tsinakis, A survey of residuated lattices. In J. Martínez (eds), Ordered Algebraic Structures, 19-56. Kluwer, Dordrecht 2002.

[11] J. A. Kalman, Lattices with involution. Trans. Amer. Math. Soc. 87 (1958), 485-491.

[12] M. Ward and R. P. Dilworth, Residuated lattices. Trans. Amer. Math. Soc. 45 (1939), $335-354$.

Authors' addresses:

Ivan Chajda

Palacký University Olomouc

Faculty of Science

Department of Algebra and Geometry

17. listopadu 12

77146 Olomouc

Czech Republic

ivan.chajda@upol.cz

Miroslav Kolařík

Palacký University Olomouc

Faculty of Science

Department of Computer Science

17. listopadu 12

77146 Olomouc

Czech Republic

miroslav.kolarik@upol.cz

Helmut Länger

TU Wien

Faculty of Mathematics and Geoinformation

Institute of Discrete Mathematics and Geometry

Wiedner Hauptstraße 8-10

1040 Vienna

Austria, and

Palacký University Olomouc

Faculty of Science

Department of Algebra and Geometry

17. listopadu 12

77146 Olomouc 
Czech Republic

helmut.laenger@tuwien.ac.at 